
\title{
$\begin{array}{ll}\text { Research Square } & \text { Preprints are preliminary reports that have not undergone peer review. } \\ \text { They should not be considered conclusive, used to inform clinical practice, } \\ \text { or referenced by the media as validated information. }\end{array}$
}

\section{Can a Multitiered Copayment System Affect People's Healthcare-seeking Behavior? A Case Study of Wenzhou, China}

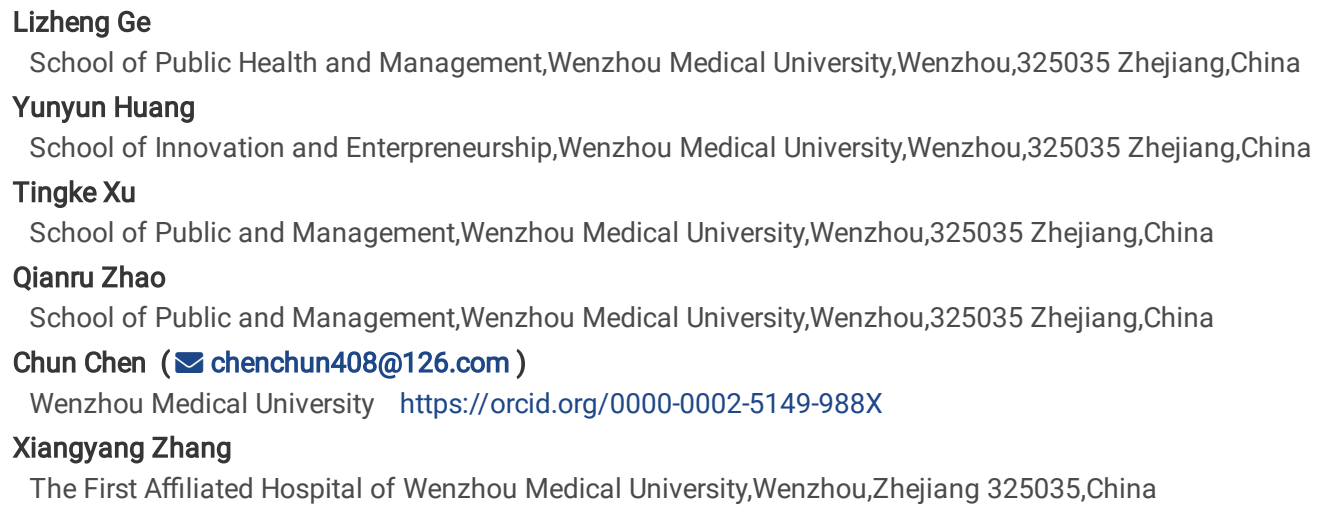




\section{Abstract}

Background. Facilitating the primary health care (PHC) system and maintaining people's reasonable health-seeking behavior are key to establishing a sustainable healthcare system. China has employed a multitiered copayment system to incentivize the public to utilize PHC services through its hierarchical medical care system; however, most people still prefer visiting tertiary care hospitals.

Methods. This preliminary study was conducted in 2016 on 1831 individuals from four regions of Wenzhou in Zhejiang Province to explore the effect and influencing factors of the multitiered copayment system that drives their primary healthcare-seeking behavior. We question whether a quality gap in healthcare services influences the minimal effect of the multitiered copayment system, which existing literature has considered as an important factor in the lack of reform in the Chinese healthcare system. We discuss the effect of the policy following the current situation in which there exists a large gap and further consider the hypothetical situation of a reduced gap in the future.

Result. The results indicate that the initial effects of the multitiered copayment system was limited. However, they become more pronounced after the equalization of the quality of healthcare services. Moreover, the main determinants in people's selection of PHC institutions changed from age and needs variables (self-rated health status) to age and enabling variables (distance to a medical care facility).

Conclusion. This study confirms that changes in the quality gap in healthcare services influence the multitiered copayment system. Hence, reducing this gap can help to achieve the intended outcome of the tiered healthcare insurance schedule.

\section{Introduction}

With a series of healthcare system reforms, China is committed to establishing a sustainable healthcare system through strengthening its primary health care (PHC) system. Reforms to strengthening PHC system are emphasized in the "Healthy China in 2030"'s blueprint and the 13th 5-year plan for medical care system reform [1]. Among these reforms, one of the most difficult to implement is the hierarchical medical care system (HMS), which was announced in 2015 [2]. China has a three-tier hospital system: tertiary, secondary, and primary hospitals [3]. The HMS means the classification of diseases according to their severity and the difficulty of treatment, then different tiers of hospitals provide different levels of healthcare services [2]. For example, tertiary hospitals provide care to emergency, severe, difficult, or complicated cases; while secondary hospitals care for the patients in acute convalescence referred from tertiary hospitals, patients in postoperative convalescence, and patients who are critically ill but in stable condition. PHC institutions mainly deal with common diseases.

A hospital's tier classification is usually determined by its function [4] and not necessarily by the quality of the healthcare services that it provides. However, due to the shortage of medical facilities, inadequate comprehensive technical support, and inappropriate financial incentives [5], PHC institutions offer a suboptimal quality of healthcare services as the healthcare institutions for common diseases [6]. Since the allocation of medical resources leans toward higher tier hospitals, it has resulted in a large gap in the quality of healthcare services provided in China. Hence, the current PHC institutions are unable to provide quality healthcare services in accordance with what is provided in the secondary and tertiary hospitals. In particular, there exists a low barrier in terms of patients' entry to higher tier hospitals, since people can directly visit higher tier hospitals without a referral [7]. As a result, to receive better treatment, patients in China prefer consulting a doctor in a secondary or tertiary tier hospital irrespective of the level of severity of their disease [8], even though the higher tier hospitals are more crowded with longer waiting times, and cost more than lower tier hospitals (healthcare services pricing policies in China allow higher tier hospitals to charge a higher price for the same services [9]).

Therefore, the promotion of the HMS has become a significant problem in Chinese medical reform, which is hindered the process of establishing a sustainable health system. In recent years, China has resorted to using health insurance, including the multitiered copayment system, as the key policy to promote the HMS. The multitiered copayment system refers to reduce the copayment rates for patients visiting PHC institutions, and the higher tier hospitals with a referral, but there is no change for patients who have direct access to the higher tier hospitals without a referral [10]. This means the system of differential deductible and copayment rates at different tiers of hospitals for inpatient and outpatient services, with the lowest copayment rate in the PHC institutions [7].

To date, the implementation of the multitiered copayment system has had little effect [11]; the higher tier hospitals are still the first choice for most patients. In 2015, the number of visits in tertiary hospitals and PHC institutions was approximately 1.50 billion and 0.21 billion, respectively [12]. However, in 2019, the number of visits in tertiary hospitals was as high as 2.05 billion, an increase of $37.7 \%$ compared to 2015 ; however, in PHC institutions it was only 0.22 billion, an increase of $11.7 \%$ compared to 2015 [12]. In 2019, there were approximately tenfold more visits to tertiary hospitals than to PHC institutions [12]. Statistically, the multitiered copayment system has not been successful in promoting people's primary healthcare-seeking behavior (See Table 1 ).

Table 1

Number of visits in different tiers of hospitals from 2015 to 2019 (unit: billion)
\begin{tabular}{|lllllll|}
\hline Number of visits \\
\hline Tiers & In $\mathbf{2 0 1 5}$ & In $\mathbf{2 0 1 6}$ & In 2017 & In $\mathbf{2 0 1 8}$ & In 2019 \\
\hline Tertiary & 1.497646 & 1.627848 & 1.726425 & 1.854787 & 2.057012 \\
\hline Secondary & 1.172331 & 1.216665 & 1.267851 & 1.284934 & 1.343425 \\
\hline Primary & 0.205679 & 0.217909 & 0.222173 & 0.224644 & 0.229652 \\
\hline Source: China health statistical yearbook 2020 [12]. & & \\
\hline
\end{tabular}


To achieve the goal of establishing a sustainable healthcare system, the Chinese government will continuously adopt the multitiered copayment system. Thus, there is important to understand why the multitiered copayment system has not changed people's health-seeking behavior. Existing literature has suggested that the gap in the quality of healthcare services is an important reason for the lack of Chinese medical reform [13], thus, we question whether the minimal effect of the multitiered copayment system is actually caused by the gaps in quality of healthcare. We conduct a preliminary study to analyze the influencing factors in the effect of the multitiered copayment system in two situations, namely: 1) the current situation of the large gap in the quality of healthcare services; and 2) the hypothetical situation of a reduced gap in the quality of healthcare services in the future.

\section{Materials And Methods}

\subsection{Data source}

While the multitiered copayment system has been implemented throughout China, the cost-sharing schemes were not the same in different regions of China. The basic healthcare insurance policy in China includes Urban Employee Basic Medical Insurance (UEBMI) and Urban Resident's Basic Medical Insurance (URBMI). For example, the URBMI policy was implemented in the city of Wenzhou, Zhejiang Province. According to data in 2016, the copayment rate for outpatient services in $\mathrm{PHC}$ institution was $50 \%, 60 \%$ in secondary hospitals, and $75 \%$ in tertiary hospitals. For inpatient services, the copayment rate was $10 \%$ in PHC institution, and up to $20 \%$ in secondary hospitals. If the patients went to a tertiary hospital, their copayment rate would be increased to $25 \%$ (See Table 2).

Table 2

Different copayment rate for outpatient and inpatient services

\begin{tabular}{|lll|}
\hline \multicolumn{2}{|l|}{ Copayment rate } \\
\hline Tiers & Outpatient services & Inpatient services \\
\hline Tertiary & $75 \%$ & $25 \%$ \\
\hline Secondary & $60 \%$ & $20 \%$ \\
\hline Primary & $50 \%$ & $10 \%$ \\
\hline
\end{tabular}

This study used a multistage stratified cluster random sampling method. The data was collected in 2016, and the sample was selected from four regions of Wenzhou: Ouhai, Ruian, Yongjia, and Taishun. Based on the level of economic development in the sampling areas, we selected one town and one county from each region; then, four representative streets/towns were selected from each town/county; and two committees/villages were selected from each street/town as the cross-sectional survey samples. Finally, we randomly selected 30 households per community as our sample for investigation.

The inclusion criteria for respondents were: (1) Individuals over the age of 15. (2) Individuals who knew the details of the survey content and agreed to participate. (3) Individuals who were able to express their ideas clearly and voluntarily.

The exclusion criteria for respondents were: (1) Individuals with visual impairment. (2) Individuals with delirium, dementia, and other mental disorders and those have no interest to participate.

There were 960 households in 4 regions (including Ouhai, Ruian, Yongjia, Taishun), 10 streets, and 6 towns in the sample. A total of 1854 questionnaires were sent out, and 1831 of the responses were valid.

\subsection{Explanatory variables}

\subsubsection{Dependent variable}

The dependent variables included whether offering the multitiered copayment system would influence the public's PHC institutions' selections in two situations, including: 1) the current situation of the large gap in the quality of healthcare services and 2) the hypothetical situation of a reduced gap in the quality of healthcare services in the future.

The following questions were asked: Does the current offering of the multitiered copayment system affect your PHC institutions' selections? Moreover, under the hypothetical situation of a reduced gap in the quality of healthcare services, will the offer of the multitiered copayment system still influence your PHC institution's selection? The alternative responses were "have effect," "no effect," and "uncertainty."

\subsubsection{Independent variable}

The Anderson model is a theoretical framework that has been fully verified and recognized, which aimed to understand the determinants of healthcare services utilization [14]. According to the Anderson model, independent variables were examined across three dimensions: predisposing variables, enabling variables, and need variables.

Predisposing variables: represent whether people's healthcare needs and social status affect their health-seeking behavior [15-17]. The predisposing variables in this study include age, education, marital status, and employment status. 
Enabling variables: represent financial and social factors that affect people's health-seeking behavior [15]. The enabling variables in this paper include social support, types of insurance, distance to a medical care facility, and household income. Enabling variables were assessed by asking respondents if they could get help from others (such as family, friends, colleagues, neighbors) when they needed it. There were three possible responses: "absolutely," "occasionally," and "not at all." And based on their responses, we classified their social support level as "good," "medium," or "poor." Then by asking respondents' household income in 2015. As shown in Table 3, we distinguished four regions (Ouhai, Ruian, Taishun, Yongjia), and we also collapsed these data into quartiles for analyses. (Q1/4= "poor," Q2/4 = "medium," Q3/4 = "good.")

Table 3

Four regions respondents' household income quartile partition table in 2015 (unit: yuan)

\begin{tabular}{|c|c|c|c|}
\hline \multicolumn{4}{|c|}{ Household Income } \\
\hline Region & Q1/4(Poor) & Q2/4(Medium) & Q3/4(Good) \\
\hline Ouhai & $<40,000$ & $40,000-100,000$ & $>100,000$ \\
\hline Ruian & $<50,000$ & $50,000-130,000$ & $>130,000$ \\
\hline Taishun & $<35,000$ & $35,000-100,000$ & $>100,000$ \\
\hline Yongjia & $<40,000$ & $40,000-100,000$ & $>100,000$ \\
\hline \multicolumn{4}{|c|}{${ }^{*}$ Good means a household's income is high in their area } \\
\hline \multicolumn{4}{|c|}{ *Medium means a household's income is medium in their are } \\
\hline \multicolumn{4}{|c|}{ *Poor means a household's income is low in their area } \\
\hline
\end{tabular}

Needs variables: represent people's perception of their general health, functional status and how the severity of their diseases affected their health-seeking behavior [15]. The needs variables in this paper include types of chronic diseases and self-rated health status. The self-rated health status score was the respondents' assessment of their health status on the day of the survey. The health score ranged from 0 to 100 ; a score $\geq 90$ was classified as "good," $\leq 70$ as "poor," and 71-89 as "medium."

\subsubsection{Time variable}

It is generally accepted that a large gap exists in healthcare services between the higher tier hospitals and PHC institutions in China. Thus, we defined the existing gaps in the quality of healthcare services between the higher tier hospitals and PHC institutions as a pre-change phase, and the hypothetical situation of a reduced gap in the quality of healthcare services in the future between the higher tier hospitals and PHC institutions as a post-change phase.

\subsection{Statistics analysis}

We used SPSS version 26 for our analysis. We first conducted a descriptive statistical analysis to introduce the effect of the multitiered copayment system pre- and post-change. Then we used chi-square analysis and Fisher's exact test to examine the difference of variables. After Fisher's exact test and chi-square analysis of the above variables, the variables associated with $p=0.2$ and below were entered in a multinomial logistic regression model to explore the factors affecting people's $\mathrm{PHC}$ institutions' selections at pre- and post-change phase [18].

\section{Results}

\subsection{Basic characteristics}

There were 1831 respondents in the study. In which $51.4 \%$ were men and $48.6 \%$ were women. The average age of the respondents was $50.371 \pm 14.940$. In terms of education level, $59 \%$ had a primary or secondary school education, $13.3 \%$ had a high school or technical school education, and $10.8 \%$ had college or higher education. As enabling variables, their average household income was 9,7976 $\pm 15,9827$ yuan. Most (81.4\%) of the respondents lived near a PHC institution within 10 minutes, according to their usual mode of transportation. Among the need variables, the respondents' average scores of self-rated health status were $78.917 \pm 12.7297$ (Table 4).

\subsection{The impact of the multitiered copayment system on the respondents' primary healthcare-seeking behavior at pre- and post-change phase}

In terms of the impact of the multitiered copayment system, at the pre-change phase, among respondents who thought the multitiered copayment system was effective, the proportion of those aged $15-24$ years was $0.8 \%$; $25-34$ years, $2.8 \%$; $35-44$ years, $3.2 \%$; $45-54$ years, 3.7\%; $54-64$ years, $1.9 \%$; and more than 65 years, $1.7 \%$. This age variable was statistically significant $(p<0.001)$. In addition, the variables of marital status, employment status, social support, and types of insurance all had statistical significance $(p<0.05)$ (Table 4$)$.

At the post-change phase, among the respondents who thought the policy was effective, the proportion of those aged 15-24 years increased to $1.5 \%$; $25-34$ years, to $7.6 \%$; $35-44$ years, to $12.3 \%$; $45-54$ years, to $12.8 \%$; $54-64$ years, to $8.7 \%$; and older than 65 years, to $7.9 \%$, with statistically significant differences $(p<0.001)$. Moreover, the variables of education, marital status, employment status, and social support were all statistically significant $(p<0.001)$. Regarding 
the variable of distance to a medical care facility, the proportion of respondents could reach at a PHC institution within 10 minutes was $42.0 \%$, and that of respondents could reach at a PHC institution within 20 minutes was $8.7 \%$, with statistically significant differences $(p<0.001)$. The variable of household income was statistically significant $(p<0.05)$. At this phase, the number of respondents who believed that the multitiered copayment system had an impact on their PHC institutions' selection increased (Table 4). 
Table 4

Characteristic of 1831 people of 2016( $\mathrm{N}=1831)$

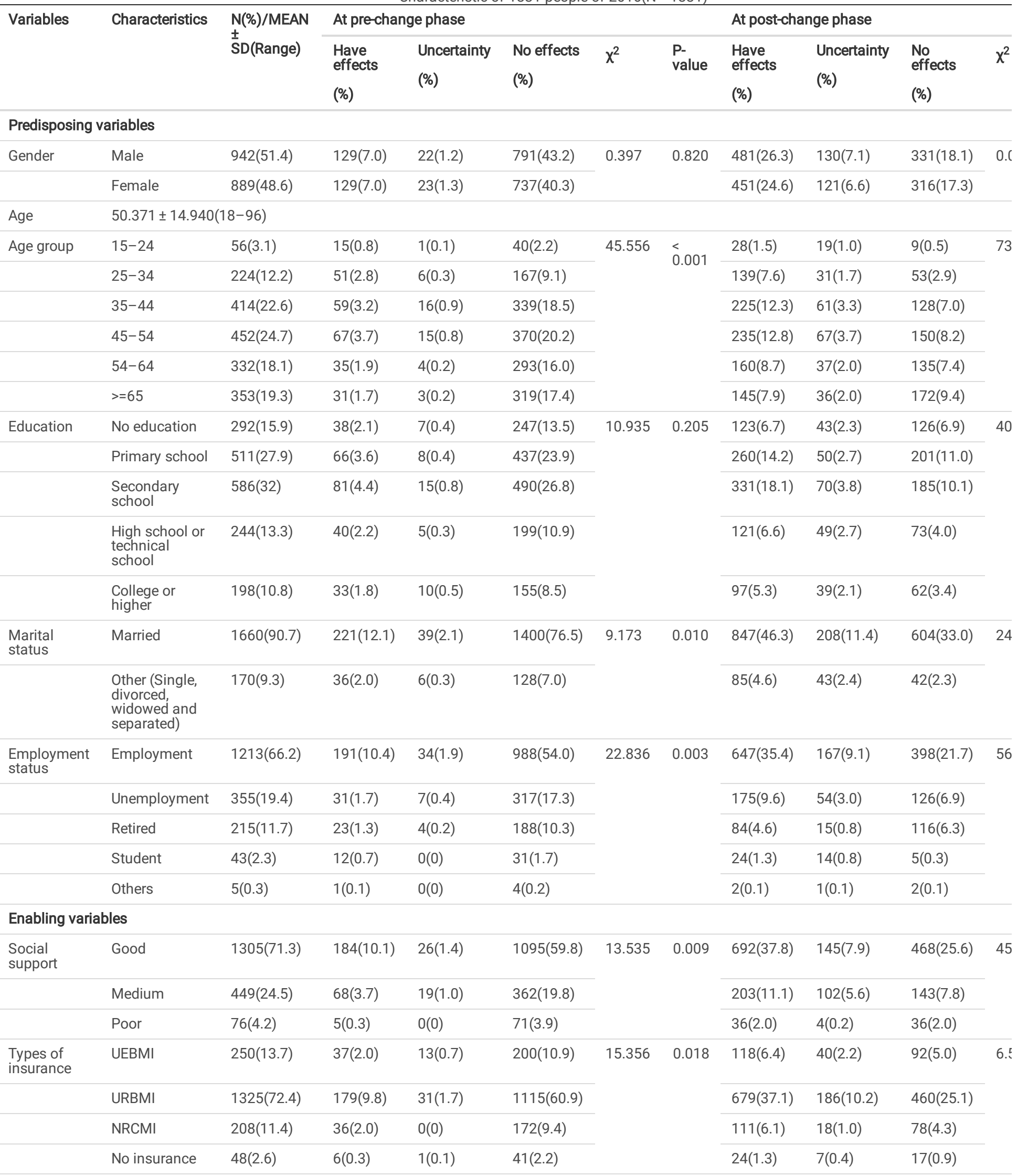

*UEBMI: Urban Employee Basic Medical Insurance

*URBMI: Urban Resident's Basic Medical Insurance

*NRCMI: New Rural Cooperative Medical Insurance 


\begin{tabular}{|c|c|c|c|c|c|c|c|c|c|c|c|}
\hline \multirow[t]{2}{*}{ Variables } & \multirow[t]{2}{*}{ Characteristics } & \multirow{2}{*}{$\begin{array}{l}\text { N(\%)/MEAN } \\
\pm \text { SD(Range) }\end{array}$} & \multicolumn{5}{|c|}{ At pre-change phase } & \multicolumn{4}{|c|}{ At post-change phase } \\
\hline & & & $\begin{array}{l}\begin{array}{l}\text { Have } \\
\text { effects }\end{array} \\
\text { (\%) }\end{array}$ & $\begin{array}{l}\text { Uncertainty } \\
(\%)\end{array}$ & $\begin{array}{l}\text { No effects } \\
(\%)\end{array}$ & $x^{2}$ & $\begin{array}{l}P- \\
\text { value }\end{array}$ & $\begin{array}{l}\begin{array}{l}\text { Have } \\
\text { effects }\end{array} \\
\text { (\%) }\end{array}$ & $\begin{array}{l}\text { Uncertainty } \\
(\%)\end{array}$ & $\begin{array}{l}\text { No } \\
\text { effects } \\
\text { (\%) }\end{array}$ & $x^{2}$ \\
\hline \multirow[t]{3}{*}{$\begin{array}{l}\text { Distance to } \\
\text { a medical } \\
\text { care facility }\end{array}$} & $\begin{array}{l}\text { Arrive within } \\
10 \text { minutes } \\
\text { (inclusive) }\end{array}$ & $1490(81.4)$ & 201(11.6) & $35(2.0)$ & $1254(72.5)$ & 4.138 & 0.244 & $725(42.0)$ & 208(12.0) & $556(32.2)$ & 50 \\
\hline & $\begin{array}{l}\text { Arrive within } \\
20 \text { minutes } \\
\text { (inclusive) }\end{array}$ & $219(12)$ & $33(1.9)$ & $6(0.3)$ & $180(10.4)$ & & & $150(8.7)$ & $31(1.8)$ & $38(2.2)$ & \\
\hline & $\begin{array}{l}\text { Arrive more } \\
\text { than } 20 \\
\text { minutes }\end{array}$ & $20(1.1)$ & $0(0)$ & $1(0.1)$ & 19(1.1) & & & $2(0.1)$ & $4(0.2)$ & $14(0.8)$ & \\
\hline $\begin{array}{l}\text { Household } \\
\text { Income }\end{array}$ & \multicolumn{11}{|c|}{$9.7976 \pm 15.9827(0-500)$} \\
\hline \multirow{3}{*}{$\begin{array}{l}\text { Household } \\
\text { Income }\end{array}$} & Good & $395(21.6)$ & $62(3.4)$ & $11(0.6)$ & $322(17.6)$ & 2.383 & 0.666 & 188(10.3) & $64(3.5)$ & $143(7.8)$ & 11 \\
\hline & Medium & 1058(57.8) & $148(8.1)$ & $27(1.5)$ & $883(48.2)$ & & & $571(31.2)$ & $128(7.0)$ & $358(19.6)$ & \\
\hline & Poor & $378(20.6)$ & $48(2.6)$ & $7(0.4)$ & $323(17.6)$ & & & $173(9.5)$ & $59(3.2)$ & 146(8.0) & \\
\hline \multicolumn{12}{|c|}{ Need variables } \\
\hline \multirow{6}{*}{$\begin{array}{l}\text { Types of } \\
\text { chronic } \\
\text { diseases }\end{array}$} & 0 & $1131(61.8)$ & $175(9.6)$ & $26(1.4)$ & $930(50.8)$ & 10.675 & 0.272 & $574(31.4)$ & 166(9.1) & $390(21.3)$ & 15 \\
\hline & 1 & $463(25.3)$ & $59(3.2)$ & $13(0.7)$ & $391(21.4)$ & & & $246(13.4)$ & $59(3.2)$ & 158(8.6) & \\
\hline & 2 & $176(9.6)$ & $15(0.8)$ & $6(0.3)$ & $155(8.5)$ & & & $84(4.6)$ & $16(0.9)$ & $76(4.2)$ & \\
\hline & 3 & $48(2.6)$ & $6(0.3)$ & $0(0)$ & $42(2.3)$ & & & $22(1.2)$ & $8(0.4)$ & $18(1.0)$ & \\
\hline & 4 & $12(0.7)$ & $3(0.2)$ & $0(0)$ & $9(0.5)$ & & & $6(0.3)$ & $1(0.1)$ & $5(0.3)$ & \\
\hline & $>=5$ & $1(0.1)$ & $0(0)$ & $0(0)$ & $1(0.1)$ & & & $0(0)$ & $1(0.1)$ & $0(0)$ & \\
\hline $\begin{array}{l}\text { Self-rated } \\
\text { health } \\
\text { status } \\
\text { scores }\end{array}$ & \multicolumn{11}{|c|}{$78.917 \pm 12.7297(1-100)$} \\
\hline \multirow{3}{*}{$\begin{array}{l}\text { Self-rated } \\
\text { health } \\
\text { status }\end{array}$} & Good & $557(30.4)$ & $91(5.0)$ & $13(0.7)$ & $453(24.7)$ & 8.238 & 0.083 & $292(16.0)$ & $73(4.0)$ & 192(10.5) & 7.6 \\
\hline & Medium & $715(39)$ & $84(4.6)$ & $14(0.8)$ & 617(33.7) & & & $343(18.7)$ & $114(6.2)$ & $257(14.0)$ & \\
\hline & Poor & $559(30.5)$ & $83(4.5)$ & $18(1.0)$ & $458(25.0)$ & & & $297(16.2)$ & $64(3.5)$ & 198(10.8) & \\
\hline \multicolumn{12}{|c|}{ *UEBMI: Urban Employee Basic Medical Insurance } \\
\hline \multicolumn{12}{|c|}{ *URBMI: Urban Resident's Basic Medical Insurance } \\
\hline
\end{tabular}

\subsection{Factor analysis of respondents' primary care-seeking behavior at pre- and post-change phases}

Table 5 presents the results of the multinomial logistic regression analysis, which analysis the factors that affect their PHC institutions' selection pre- and post-change. Respondents who considered that narrowing the gap in the quality of healthcare services between PHC institutions and the higher tier hospitals would lead to a change in their primary health-seeking behavior were used as the reference group. The impact of the multitiered copayment system is reflected in the respondents' selections of PHC institutions.

At the pre-change phase, the results indicated that age and self-rated health status were the main influencing factors. Compared with the respondents aged over 65 years, age groups of 25-34 and 45-54 were less vulnerable to policy and selected PHC institutions. It is evident that older respondents are typically more likely to be affected by the multitiered copayment system when compared to younger respondents. In addition, in comparison to respondents with poor self-rated health status, respondents with moderate self-rated health status are more sensitive to the incentive of the multitiered copayment system (Table 5).

At the post-change phase, respondents in the age ranges of $24-34,35-44$, and $45-54$ are not as susceptible to the multitiered copayment system as those over 65 years, and it also shows a trend in the pre-change phase. Compared with those who lived more than 20 minutes away from a PHC institution, respondents who lived within 10 and 20 minutes of a PHC institution are less susceptible to the multitiered copayment system (Table 5).

Page $7 / 11$ 
Table 5

Multinomial logistic regression: Factors affecting people's health-seeking behavior at pre- and post-change phase

\begin{tabular}{|c|c|c|c|c|c|}
\hline \multirow[t]{2}{*}{ Variables } & \multirow[t]{2}{*}{ Categories } & \multicolumn{2}{|c|}{$\begin{array}{l}\text { No effect (Model 1) Reference } \\
\text { group = Have effects }\end{array}$} & \multicolumn{2}{|c|}{$\begin{array}{l}\text { No effect (Model 2) Reference } \\
\text { group = Have effects }\end{array}$} \\
\hline & & OR $(95 \% \mathrm{Cl})$ & p-value & OR $(95 \% \mathrm{Cl})$ & p-value \\
\hline Intercept & & & 0.437 & & 0.007 \\
\hline \multicolumn{6}{|l|}{ Predisposing variables } \\
\hline \multirow[t]{5}{*}{ Age $(\operatorname{Ref}=>=65)$} & $15-24$ & $2.730(0.859-8.678)$ & 0.089 & $1.264(0.344-4.642)$ & 0.724 \\
\hline & $25-34$ & $2.732(1.548-4.824)$ & 0.001 & $2.619(1.561-4.394)$ & $<0.001$ \\
\hline & $35-44$ & $1.605(0.923-2.793)$ & 0.094 & $1.794(1.172-2.746)$ & 0.007 \\
\hline & $45-54$ & $1.778(1.047-3.019)$ & 0.033 & $1.566(1.072-2.289)$ & 0.020 \\
\hline & $54-64$ & $0.435(0.723-2.125)$ & 0.435 & $1.288(0.900-1.844)$ & 0.166 \\
\hline \multicolumn{6}{|l|}{ Need variables } \\
\hline \multirow[t]{2}{*}{ Self-rated health status (Ref = Poor) } & Good & $0.950(0.676-1.334)$ & 0.766 & - & - \\
\hline & Moderate & $0.709(0.507-0.992)$ & 0.045 & - & - \\
\hline \multicolumn{6}{|l|}{ Enabling variables } \\
\hline \multirow[t]{2}{*}{$\begin{array}{l}\text { Distance to a medical care facility (Ref }=\text { Arrive } \\
\text { more than } 20 \text { minutes) }\end{array}$} & $\begin{array}{l}\text { Arrive within } 10 \\
\text { minutes (inclusive) }\end{array}$ & - & - & $11.643(2.559-52.978)$ & 0.001 \\
\hline & $\begin{array}{l}\text { Arrive within } 20 \\
\text { minutes (inclusive) }\end{array}$ & - & - & $33.215(7.025-157.051)$ & $<0.001$ \\
\hline \multicolumn{6}{|l|}{ Model 1 means at pre-change phase. } \\
\hline Model 2 means at post-change phase. & & & & & \\
\hline
\end{tabular}

\section{Discussion}

By studying the effects of the multitiered copayment system, our findings demonstrate that its introduction had different effects at the pre- and post-change phase. Compared with the pre-change phase, the number of respondents who believed the multitiered copayment system had an impact on their PHC institutions' selection at the post-change phase had a threefold increase (from $14.09-50.90 \%$ ), and the multitiered copayment system started to play a positive role.

At the pre-change phase, owing to the large gaps in the quality of healthcare services, and the small gap in the copayment rate in different tiers of hospitals, the effect of the reduction in the multitiered copay rate on people's primary healthcare-seeking behavior was limited. This result corresponds with the results from a numerical experiment which concluded that in order to affect people's primary healthcare-seeking behavior, the optimal copayment difference should be large enough, such as $37 \%$ in tertiary hospitals and $4 \%$ in PHC institutions [11]. However, such a gap in the copayment rate would increase health and healthcare inequity. It was a challenge to increase the effectiveness of the multitiered copayment system by adjusting the copayment rate at the pre-change phase. Therefore, the multitiered copayment system failed to show a meaningful effect in the significant gap of quality in healthcare services.

The results of the multinomial logistic regression analysis, at the pre-change phase, indicate that the age of the respondents and their needs factor of selfrated health status are the main determinants of their PHC institutions' selections. Further, these factors have also been proven in previous studies [19, 20]. Age has been identified as the most common variable, which is similar in our study as well. We found that older people are more likely to change their primary healthcare-seeking behavior due to the copayment rate reduction. This may be because the older adults are most likely to have chronic diseases that need continuous care [20], and the diagnosis and treatment of chronic disease are highly standardized. In other words, the services required by older adults do not usually require advanced technology and equipment. Meanwhile, people with chronic conditions often report moderate or poor self-rated health status [20], which means that there is a sizable overlap between the elderly and those with moderate and poor self-rated health status [21]. However, those with poor selfrated health scores tend to believe that they have more complicated disease, need higher levels of treatment, and that the services in PHC institutions cannot meet their medical care needs. Thus, due to their higher frequency of hospital visit and lower needs in diagnosis and treatment, the elderly and those with moderate self-rated health status would be motivated by the copayment rate reduction in PHC institutions.

As mentioned above, the effect of the multitiered copayment system will be improved at the post-change phase. The role of the gap in the quality of healthcare services in PHC institutions was found in South Korea (non-equalization in services quality) and the United Kingdom (UK) (even equalization in services quality). In the UK, the general practitioner system is the focal point of PHC [22]. And the UK's National Health Service attach great importance to the quality of their healthcare service [23]. Thus, the key to system effectiveness is that the general practitioners always provide high quality of healthcare services [24]. However, in Korea, in order to guide patients with mild disease to PHC institutions, a copayment policy was implemented [25]. In this policy, the copay rates for outpatients are $30 \%$ in clinics and pharmacies, $40 \%$ in hospitals, $50 \%$ in general hospitals, and $60 \%$ in tertiary hospitals [26]. However, the result of this copayment policy in Korea had little effect [27, 28], which was consistent with the findings from other studies in China, and ours. As the quality of healthcare services in Korea is similar to that of China's, which also has a large gap between the higher tier hospitals and those PHC institutions, allocation of 
medical care resources also tended to be biased towards higher tier hospitals. Thus, patients consider physicians in tertiary hospitals to be more qualified and prefer to choose these highly qualified physicians for healthcare, which resulted in the unsatisfactory effect from the multitiered copayment system [28]. All

these evidences show that the gap in quality of healthcare services is the key factor that determines the effectiveness of the multitiered copayment system.

At the post-change phase, age and the enabling factor of distance to a medical care facility, transformed from needs factor (self-rated health status), were the critical factors affecting people's PHC institutions' selection. At this phase, people no longer consider the gap in healthcare services quality, because irrespective of the type of hospitals they visit, they would receive the same quality of treatment. Thus, the convenience of healthcare access is the primary factor for them to consider. In general, PHC institutions are closer to people's homes, which makes it more convenient for people to receive healthcare services. Additionally, PHC institutions offer better coverage rate, shorter wait times, and located in residential areas, healthcare workers there may be familiar with patients, which will make it easier to receive targeted healthcare services [29]. In this case, not only the elderly or people with moderate self-rated health status, but also others with healthcare needs can benefit from the reduced copay to receive care; thus, more people would visit those PHC healthcare facilities.

\section{Limitation}

This preliminary study has the following limitations. First, our samples are limited to Wenzhou, which is a medium-sized city in Zhejiang Province, southeastern China. Second, the results were based on respondents' answers to our questionnaire, which were their subjective opinions rather than objective measures.

Thus, the scope of study needs to be expanded in the future, and studies about the specific objective measure of the multitiered copayment system must be conducted, so that local governments can obtain more accurate information, adjust measures according to particular local conditions, and achieve optimal outcome from the policy. Finally, future study is also required regarding the issue of the public's willingness to pay for healthcare.

\section{Conclusions}

The multitiered copayment system in Wenzhou has little effect so far. Among all, we found older adults and those with moderate self-rated health status are the main groups that are more likely to be affected by this policy. The low quality of care in PHC institutions in Wenzhou reduced the effectiveness of the multitiered copayment schedule's intended outcome. In the context of the equalized quality of healthcare services in all tiers of healthcare facilities, the effect of the multitiered copayment system can be enhanced. Considering the factors of higher cost, long waiting time, and further distance from home, seeking care at higher tier hospitals is not the best choice for most people. Therefore, narrowing the gap in the quality of healthcare services among different tiers of hospitals is crucial in Wenzhou, as well as in other areas of China. In order to promote a sustainable healthcare system, more PHC healthcare institutions should be established at the community level. Meanwhile, the key aspect of making the multitiered copayment system effective remains to be the improvement of the quality of healthcare services.

\section{List Of Abbreviations}

PHC (Primary Health Care)

\section{Declarations}

\section{Ethics approval and consent to participate}

This study was approved by the ethics committee at Wenzhou Medical University (approval number 2016-052).

\section{Consent for publication}

Not applicable.

\section{Availability of data and materials}

The datasets used and analysed during the current study are available from the corresponding author on reasonable request.

\section{Competing interests}

The authors declare that they have no competing interests.

\section{Funding}

This project was sponsored by the National Natural Science Foundation of China (71603187), Zhejiang Provincial Natural Science Foundation (LQ16G030011), and the Key Social Science Project for University Teachers of Zhejiang, China (2014QN005).

\section{Authors' contributions}

LG is responsible for conceptualization, data curation, investigation, methodology, resources, software, and writing of the original draft.

$\mathrm{YH}$ is responsible for data curation, investigation, methodology, resources, software. 
TX is responsible for methodology, resources, software.

QZ is responsible for data curation, investigation, software.

$\mathrm{CC}$ is responsible for conceptualization, funding acquisition, resources, supervision, and reviewing and editing the manuscript.

$\mathrm{XZ}$ is responsible for investigation, and reviewing and editing the manuscript

\section{Acknowledgements}

The authors thank Zuokai Xie and Qingsong Wu for their valuable assistance.

\section{References}

1. Li, L.; Fu, H. China's health care system reform: Progress and prospects. Int J Health Plann Manage 2017, 32, 240-253, doi:10.1002/hpm.2424.

2. Zhou, Z.; Zhao, Y.; Shen, C.; Lai, S.; Nawaz, R.; Gao, J. Evaluating the effect of hierarchical medical system on health seeking behavior: A difference-indifferences analysis in China. Soc Sci Med 2020, 268, 113372, doi:10.1016/j.socscimed.2020.113372.

3. Wang, J.; Wang, P.; Wang, X.; Zheng, Y.; Xiao, Y. Use and prescription of antibiotics in primary health care settings in China. JAMA internal medicine 2014, $174,1914-1920$.

4. Li, L.; Du, T.; Hu, Y. The effect of different classification of hospitals on medical expenditure from perspective of classification of hospitals framework: evidence from China. Cost Effectiveness and Resource Allocation 2020, 18, 1-12.

5. Li, X.; Lu, J.; Hu, S.; Cheng, K.K.; De Maeseneer, J.; Meng, Q.; Mossialos, E.; Xu, D.R.; Yip, W.; Zhang, H.; et al. The primary health-care system in China. Lancet 2017, 390, 2584-2594, doi:10.1016/S0140-6736(17)33109-4.

6. Li, X.; Krumholz, H.M.; Yip, W.; Cheng, K.K.; De Maeseneer, J.; Meng, Q.; Mossialos, E.; Li, C.; Lu, J.; Su, M.; et al. Quality of primary health care in China: challenges and recommendations. Lancet 2020, 395, 1802-1812, doi:10.1016/S0140-6736(20)30122-7.

7. Mossialos, E.; Wenzl, M.; Osborn, R.; Sarnak, D. 2015 international profiles of health care systems; Canadian Agency for Drugs and Technologies in Health: 2016.

8. Liu, Y.; Kong, Q.; Yuan, S.; van de Klundert, J. Factors influencing choice of health system access level in China: A systematic review. PLoS One 2018, 13, e0201887, doi:10.1371/journal.pone.0201887.

9. National Health Commission. China health and family planning statistical yearbook 2016. Beijing: Peking Union Medical College 2016.

10. Shen, M.H.; He, W.; Li, L.Y. Incentives to use primary care and their impact on healthcare utilization: Evidence using a public health insurance dataset in China. Social Science \& Medicine 2020, 255, 112981, doi:ARTN 112981 10.1016/j.socscimed.2020.112981.

11. Deng, Y.; Li, N.; Xu, X.; Jiang, Z. Differentiation copayment design to improve the multi-tiered healthcare system in China. In Proceedings of the 2017 13 th IEEE Conference on Automation Science and Engineering (CASE), 2017; pp. 201-206.

12. National Health Commission. China health statistical yearbook 2020. Beijing: Peking Union Medical College 2020.

13. Hu, S.L.; Tang, S.L.; Liu, Y.L.; Zhao, Y.X.; Escobar, M.L.; de Ferranti, D. Health System Reform in China 6 Reform of how health care is paid for in China: challenges and opportunities. Lancet 2008, 372, 1846-1853, doi:10.1016/S0140-6736(08)61368-9.

14. Anderson, J.G. Health services utilization: framework and review. Health Serv Res 1973, 8, 184-199.

15. Andersen, R.M.; Davidson, P.L.; Baumeister, S.E. Improving access to care. Changing the US health care system: key issues in health services policy and management. San Francisco: Jossey-Bass 2014, 36, 33-69.

16. Bass, D.M.; Noelker, L.S. The influence of family caregivers on elder's use of in-home services: An expanded conceptual framework. Journal of health and social behavior 1987, 184-196.

17. Hulka, B.S.; Wheat, J.R. Patterns of utilization. The patient perspective. Med Care 1985, 23, 438-460, doi:10.1097/00005650-198505000-00009.

18. Asfar, T.; Ahmad, B.; Rastam, S.; Mulloli, T.P.; Ward, K.D.; Maziak, W. Self-rated health and its determinants among adults in Syria: a model from the Middle East. BMC Public Health 2007, 7, 177.

19. Geitona, M.; Zavras, D.; Kyriopoulos, J. Determinants of healthcare utilization in Greece: implications for decision-making. Eur J Gen Pract 2007, 13, 144150, doi:10.1080/13814780701541340.

20. Park, J.M. Health status and health services utilization in elderly Koreans. Int J Equity Health 2014, 13, 73, doi:10.1186/s12939-014-0073-7.

21. Mantyselka, P.T.; Turunen, J.H.; Ahonen, R.S.; Kumpusalo, E.A. Chronic pain and poor self-rated health. JAMA 2003, 290, 2435-2442, doi:10.1001/jama.290.18.2435.

22. Boyle, S. United Kingdom (England): Health system review. Health Syst Transit 2011, 13, 1-483, xix-xx.

23. Madhok, R. Crossing the quality chasm: lessons from health care quality improvement efforts in England. In Proceedings of the Baylor University Medical Center Proceedings, 2002; pp. 77-83.

24. Toon, P.D. What is good general practice? A philosophical study of the concept of high quality medical care. Occasional paper (Royal College of General Practitioners) 1994, i.

25. Lee, H.J.; Jang, S.-I.; Park, E.-C. The effect of increasing the coinsurance rate on outpatient utilization of healthcare services in South Korea. BMC health services research 2017, 17, 152. 
26. Lee, W.Y.; Shaw, I. The impact of out-of-pocket payments on health care inequity: the case of national health insurance in South Korea. Int J Environ Res Public Health 2014, 11, 7304-7318, doi:10.3390/ijerph110707304.

27. Kim, J.; Ko, S.; Yang, B. The effects of patient cost sharing on ambulatory utilization in South Korea. Health Policy 2005, 72, 293-300, doi:10.1016/j.healthpol.2004.09.002.

28. Kim, A.M.; Cho, S.; Kim, H.J.; Jung, H.; Jo, M.-W.; Lee, J.Y.; Eun, S.J. Primary care Patients' preference for hospitals over clinics in Korea. International journal of environmental research and public health 2018, 15, 1119.

29. Wang, W.; Shi, L.; Yin, A.; Lai, Y.; Maitland, E.; Nicholas, S. Development and validation of the Tibetan primary care assessment tool. Biomed Res Int 2014, 2014, 308739, doi:10.1155/2014/308739. 